OPEN ACCESS

Edited by: Fabrizio Mattei,

National Institute of Health (ISS), Italy

Reviewed by:

Matthew S. Macauley, University of Alberta, Canada Sebastian Peter Galuska, Leibniz Institute for Farm Anima

Biology (FBN), Germany

*Correspondence:

Qing Yuan

qingyuan@swmu.edu.cn

Siji Nian

sijinian@swmu.edu.cn

Specialty section:

This article was submitted to Cancer Immunity and Immunotherapy,

a section of the journal

Frontiers in Oncology

Received: 19 September 2020 Accepted: 22 February 2021

Published: 16 March 2021

Citation:

Zhang H, Xie Y, Hu Z, Yu H, Xie X Ye Y, Xu W, Nian S and Yuan Q (2021) Integrative Analysis of the Expression of SIGLEC Family Members in Lung Adenocarcinoma via Data Mining. Front. Oncol. 11:608113 doi: 10.3389/fonc. 2021.608113

\section{Integrative Analysis of the Expression of SIGLEC Family Members in Lung Adenocarcinoma via Data Mining}

\author{
Haiyan Zhang ${ }^{1}$, Yongfei Xie ${ }^{2}$, Zhi Hu ${ }^{3}$, Hong Yu ${ }^{1}$, Xiang Xie ${ }^{1}$, Yingchun $\mathrm{Ye}^{1}$, Wenfeng $\mathrm{Xu}{ }^{1}$, \\ Siji Nian ${ }^{1 *}$ and Qing Yuan ${ }^{1 *}$ \\ ${ }^{1}$ Public Experimental Technology Center, The School of Basic Medical Science, Southwest Medical University, Luzhou, \\ China, ${ }^{2}$ Life Sciences School, Anhui Agricultural University, Hefei, China, ${ }^{3}$ Department of Thoracic Surgery, The Affiliated \\ Hospital of Southwest Medical University, Luzhou, China
}

Background: Sialic acid-binding immunoglobulin-type lectin (SIGLEC) family members are involved in regulating immune-cell activation, proliferation, and apoptosis, and they play an important role in tumor development. However, their expression and correlation with immune molecules in lung adenocarcinoma (LUAD) remain unclear.

Methods: We utilized Gene Expression Profiling Interactive Analysis, Kaplan-Meier analysis, the limma package in R/Bioconductor, the University of California Santa Cruz Cancer Genome Browser, cBioPortal, STRING, Cytoscape, DAVID, and the Tumor Immune Estimation Resource for gene and protein profiling and analyses.

Results: The results showed that SIGLEC10 and SIGLEC15 levels were upregulated in LUAD, whereas SIGLEC1, CD22 (SIGLEC2), CD33, myelin-associated glycoprotein (SIGLEC4), SIGLEC5, SIGLEC6, SIGLEC7, SIGLEC8, SIGLEC11, and SIGLEC14 levels were significantly downregulated, with their low expression associated with poor overall survival. Moreover, we observed high SIGLEC-mutation rates $(22 \%)$ in LUAD patients, with SIGLEC functions determined as primarily involved in regulating the immune response, signal transduction, inflammatory response, and cell adhesion. Furthermore, we found that SIGLEC expression was significantly correlated with immune-cell infiltration, especially macrophages, neutrophils, and dendritic cells, and highly associated with immune molecules such as CD80, CD86, CD28, B-cell-activating factor, programmed cell death 1 ligand 2, and colony stimulating factor 1 receptor.

Conclusion: These results provide insight into the potential molecular mechanism associated with SIGLEC-related development of LUAD, as well as clues for screening biomarkers and therapeutic targets.

\section{Keywords: lung adenocarcinoma, bioinformatics, SIGLEC, survival, immunotherapy}

\section{INTRODUCTION}

High cancer rates worldwide continue to attract the attention of both scientists and medical workers. In most countries, the incidence and mortality rates of lung cancer occupies the first or second position in terms of malignancy (1). Approximately $85 \%$ of lung cancer patients are diagnosed with non-small cell lung cancer (NSCLC), of which lung adenocarcinoma (LUAD) is the 
most common subtype, accounting for about $40 \%$ of primary lung tumors (2). Additionally, LUAD is the most common form of lung cancer in women, patients with no history of smoking, and people aged $<40$ years (3). LUADs usually originate in peripheral lung tissue and can maintain localization there for extended periods before symptoms appear, the more generalized early versions of which (e.g., fatigue, subtle shortness of breath, or upper back, and chest pain) can be missed or attributed to other causes.

Targeted therapies inhibit the growth of cancer cells by blocking the activity of specific oncogenic signaling molecules (4). Some genetic alterations observed in LUAD include gene mutations in epidermal growth factor receptor, BRAF, and human epidermal growth factor receptor 2, gene fusions, and rearrangements in ALK, ROS1, or RET (5-9). Drugs, such as afatinib, alectinib, ceritinib, crizotinib, erlotinib, and gefitinib, have been approved by the Food and Drug Administration for clinical use (10), and immunotherapy has rapidly grown as a major modality for LUAD treatment (11). Binding of cytotoxic T lymphocyte-associated antigen 4 (CTLA-4) to B7-1 (CD80) and B7-2 (CD86) produces an inhibitory signal that limits the production of interleukin (IL)-2 and inhibits the proliferation and activation of $\mathrm{T}$ cells. The CTLA- 4 antibody ipilimumab works to turn off this inhibitory mechanism or "release the brakes" in order to allow T cells to target and kill cancer cells (12, 13). Similar to CTLA-4, binding of programmed death-1 (PD1) by its ligand (PD-L1), which is overexpressed on the surface of some cancer cells, releases signals that inhibit $\mathrm{T}$ cell activation and promote tumor-cell evasion $(14,15)$. Monoclonal antibodies, such as pembroliz, nivolumab, and atezolizumab, target PD-1 or PD-L1 to help boost the immune response to attack and destroy cancer cells (16). However, although significant advances in lung cancer treatment have been made, the 5-year survival rate for LUAD remains low. Therefore, continued investigation of potential LUAD-related genes and identification of their mechanisms is necessary (17).

Sialic acid-binding immunoglobulin-type lectins (SIGLECs) are a family of immunomodulatory receptors and a subset of Itype lectins that can recognize sialic acid sugar-carrying glycans (sialoglycans) aberrantly expressed on many tumor cells (18). SIGLECs are characterized into two distinct groups: the first and highly conserved group comprises sialoadhesin (SIGLEC1; CD169), CD22 (SIGLEC2), myelin-associated glycoprotein (MAG; SIGLEC4), and SIGLEC15; and the second, rapidly evolving group comprises CD33-related SIGLECs, including SIGLEC3/5/6/7/8/9/10/11/14/16 (19, 20). SIGLECs regulate immune-cell signaling and are involved in pathogen recognition, modulation of immune responses, and intercellular interactions $(21,22)$. SIGLEC1 is a macrophage-restricted cell surface receptor that has been shown to contribute to sialylated pathogen uptake, lymphocyte proliferation and antigen presentation.

Abbreviations: TCGA, The Cancer Genome Atlas; PPI, protein-protein interaction; GO, Gene Ontology; KEGG, Kyoto Encyclopedia of Genes and Genomes; BP, Biological processes; CC, cellular components; MF, molecular function; DAVID, The Database for Annotation, Visualization and Integrated Discovery; TIMER, Tumor Immune Estimation Resource.
CD22 inhibits $B$ cell receptor (BCR) signaling and regulates toll-like receptor (TLR) signaling and the survival of $B$ cells. CD33, a marker of myeloid cells, plays a role in myeloid differentiation and dendritic cell maturation. MAG binds to complex gangliosides (GDla and GT1b) to inhibit axon regeneration and neurite outgrowth. It has been shown that a paired receptor system in the SIGLEC family has implications for regulation of host immunity. SIGLEC5/SIGLEC14 and SIGLEC11/SIGLEC16 represent such paired receptors, which contain similar extracellular domains and play a role in mediating host-pathogen interactions (19). SIGLEC6 is a leptin-binding protein that has functional consequences on the aberrant proliferation, apoptosis and invasion of BeWO cells (23). SIGLEC7 is the first Siglec receptor observed on human natural killer (NK) cells, SIGLEC7/SIGLEC9 ligands shield malignant cells from NK cell attack. Expression of SIGLEC7 in multiple sclerosis (MS) patients correlates with clinical course, suggesting it as a potential biomarker of acute disease activity in MS (24). Moreover, SIGLEC9 is overexpressed on tumor-associated T cells and shifts macrophages toward tumorpromoting behaviors, suggesting that targeting SIGLEC9-related pathways might improve the antitumor response (25). SIGLEC8 has been identified as a target for the treatment of eosinophil disorders. SIGLEC10 is a suppressor receptor expressed on the surface of $\mathrm{T}$ cells, which triggers immunosuppression by blocking the activation of $\mathrm{T}$ cell receptor. SIGLEC15, as a critical immune suppressor, suppresses T-cell activation and promotes the survival and differentiation of suppressor myeloid cells. SIGLEC15 not only plays a biological role in osteoclast differentiation but also in microbial infection and tumor microenvironment $(26,27)$. Furthermore, SIGLECs are abnormally expressed in a variety of malignancies, including hepatocellular carcinoma, endometrial cancer, bladder cancer, and colon cancer (26), and CD33 (28) and CD22 were identified as immunotherapeutic targets for acute myeloid leukemia and non-Hodgkin's lymphoma, respectively $(29,30)$. A recent study indicated that sialoglycan-SIGLEC interactions in the tumor microenvironment (TME) suppress effector immune-cell activity and modulate myeloid-cell functions, thereby contributing to tumor immune evasion and sustained tumor growth $(31,32)$. To our knowledge, however, there are no comprehensive reports of SIGLEC-related expression and its correlation with immune molecules in LUAD.

In this study, we comprehensively analyzed the expression of SIGLEC family members in LUAD and its relationship with patient prognosis, immune-molecule expression, and infiltrating levels of immune cells in order to expand the knowledge of SIGLEC-related regulation and function, and to promote improvements in LUAD diagnosis, treatment, and prognosis.

\section{MATERIALS AND METHODS}

\section{Gene Expression Profiling Interactive Analysis Database}

SIGLEC-expression data from 23 malignant tumors were obtained from the GEPIA database 
(http://gepia.cancer-pku.cn/), which is an interactive web application for gene-expression analysis based on RNAsequencing (RNA-seq) data from The Cancer Genome Atlas (TCGA) and Genotype-Tissue Expression databases (33). Gene-expression levels were presented using the $\log _{2}$ $(\mathrm{TPM}+1)$ scale.

\section{University of California Santa Cruz Xena Repository}

LUAD RNA-seq data were downloaded from the UCSC Cancer Genome Browser (https://genome.ucsc.edu/) (34), which contained 586 samples, including 59 normal samples and 526 tumor samples. Differential-expression analysis was performed using the $\mathrm{R}$ package limma (35), and differentially expressed genes were screened with according to a false-discovery rate $\left(\right.$ FDR) $<0.05$ and a $\mid \log _{2}$ (fold-change) $\mid>1$.

\section{Kaplan-Meier Analysis}

The correlation between individual SIGLEC family member expression and LUAD patient overall survival (OS) was analyzed using a Kaplan-Meier plotter database (https://kmplot.com/ analysis/). P-values were calculated using the log-rank test.

\section{cBioPortal Analysis}

cBioPortal (https://www.cbioportal.org/) is an open resource for exploring, analyzing, and visualizing multidimensional cancer genomics data. Data from $>5,000$ tumor samples from 20 cancer studies are currently accessible $(36,37)$. Genetic alteration and co-expression of SIGLECs were analyzed via public LUAD datasets.

\section{Protein-Protein Interaction-Network Construction}

We used Venny software (v.2.1; https://bioinfogp.cnb.csic. es/tools/venny/) to highlight which differentially expressed SIGLEC-related genes. The STRING protein interaction database (38) and Cytoscape software (39) were used to analyze and reconstruct protein-interaction networks related to SIGLECs, with the top 10 hub genes analyzed by the Cytoscape plugin cytoHubba.

\section{Gene Ontology and Kyoto Encyclopedia of Genes and Genomes Analysis}

We used the Database for Annotation, Visualization and Integrated Discovery (DAVID) bioinformatics resources version 6.8 (40) to perform functional GO and KEGG pathway enrichment analyses of genes related to SIGLEC expression. GO terms and KEGG pathways, and $p<0.05$ were considered significantly enriched.

\section{Tumor Immune Estimation Resource Analysis}

The correlation of SIGLECs with immune-cell infiltration in LUAD was analyzed by TIMER (41), which is a web-based platform that provides the abundance of six tumor-infiltrating immune subsets (B cells, $\mathrm{CD}^{+} \mathrm{T}$ cells, $\mathrm{CD}^{+} \mathrm{T}$ cells, neutrophils, macrophages, and dendritic cells) based on data from TCGA. Associations between SIGLEC expression and immune molecules were estimated by Spearman's correlation coefficient.

\section{RESULTS}

\section{SIGLECs Expression in Different Tumor Types}

First, we analyzed SIGLECs expression in various tumors via the GEPIA platform. To determine aberrant SIGLECs expression, we generated a heatmap to present the log fold change in tumor tissues relative to normal tissues. The results showed that CD22 levels was significantly upregulated in cholangiocarcinoma (CHOL) and kidney chromophobe $(\mathrm{KICH})$ cancer, and that SIGLEC1, CD22, CD33, SIGLEC7, SIGLEC8, SIGLEC9, and SIGLEC10 levels were upregulated in kidney renal clear cell carcinoma (KIRC) and kidney renal papillary cell carcinoma (KIRP) relative to levels in normal tissue. Notably, almost all SIGLEC members were downregulated in NSCLCs, such as LUAD and lung squamous cell carcinoma (LUSC) (Figure 1A).

We then focused on SIGLECs expression in LUAD as the most common histological type of lung cancer. Analysis of a LUAD-specific RNA-seq dataset revealed results consistent with previously analyzed data, and that mRNA levels of SIGLEC1, CD22, CD33, MAG, SIGLEC5, SIGLEC6, SIGLEC7, SIGLEC8, SIGLEC9, SIGLEC11, SIGLEC14, and SIGLEC16 were downregulated in LUAD tumor tissues relative to normal tissues $(p<0.001)$ (Figure 1B). SIGLEC10 and SIGLEC15 expression was also upregulated in tumor tissues, although with no significant difference relative to normal tissues.

\section{SIGLECs Are Associated With LUAD Prognosis}

To investigate the effect SIGLEC mRNA expression on LUAD prognosis, we performed Kaplan-Meier analysis. We found that decreased levels of SIGLEC1 [hazard ratio (HR) $=0.73,95 \%$ confidence interval (CI): 0.54-0.98; $p=0.037$ ], CD22 [HR = 0.63, 95\% CI: 0.47-0.84; $p=0.0017$ ], CD33 [HR $=0.56,95 \%$ CI: $0.41-0.76 ; p=0.00017], M A G$ [HR $=0.7,95 \% \mathrm{CI}: 0.52-$ $0.95 ; p=0.019$ ], SIGLEC5 [HR $=0.69,95 \%$ CI: $0.5-0.97 ; p=$ 0.032], SIGLEC6 [HR $=0.42,95 \%$ CI: $0.27-0.64 ; p=2.7 \mathrm{e}-05]$, SIGLEC7 $[\mathrm{HR}=0.68,95 \% \mathrm{CI}: 0.51-0.91 ; p=0.01]$, SIGLEC8 $[\mathrm{HR}=0.67,95 \% \mathrm{CI}: 0.5-0.9 ; p=0.037]$; SIGLEC10 [HR $=0.73$, 95\% CI: 0.54-0.99; $p=0.041$ ], SIGLEC11 [HR $=0.61,95 \% \mathrm{CI}$ : $0.45-0.81 ; p=0.00074]$, and SIGLEC16 [HR = 0.69, 95\% CI: 0.5$0.94 ; p=0.02]$ were associated with worse OS in LUAD patients, whereas SIGLEC9, SIGLEC14, and SIGLEC15 expression were not correlated with OS $(p>0.05)$ (Figure 2).

\section{cBioPortal Analysis}

We then analyzed the genetic alterations of SIGLECs based on mutation and copy number alteration data from different LUAD studies. A total of 1,832 samples were obtained from six TCGA studies, among which 403 samples (22\%) involved genetic alterations of SIGLECs [290 (15.83\%) mutations, 63 (3.44\%) amplifications, 24 (1.31\%) deep deletions, and $26(1.42 \%)$ multiple alterations] (Figure 3A). 

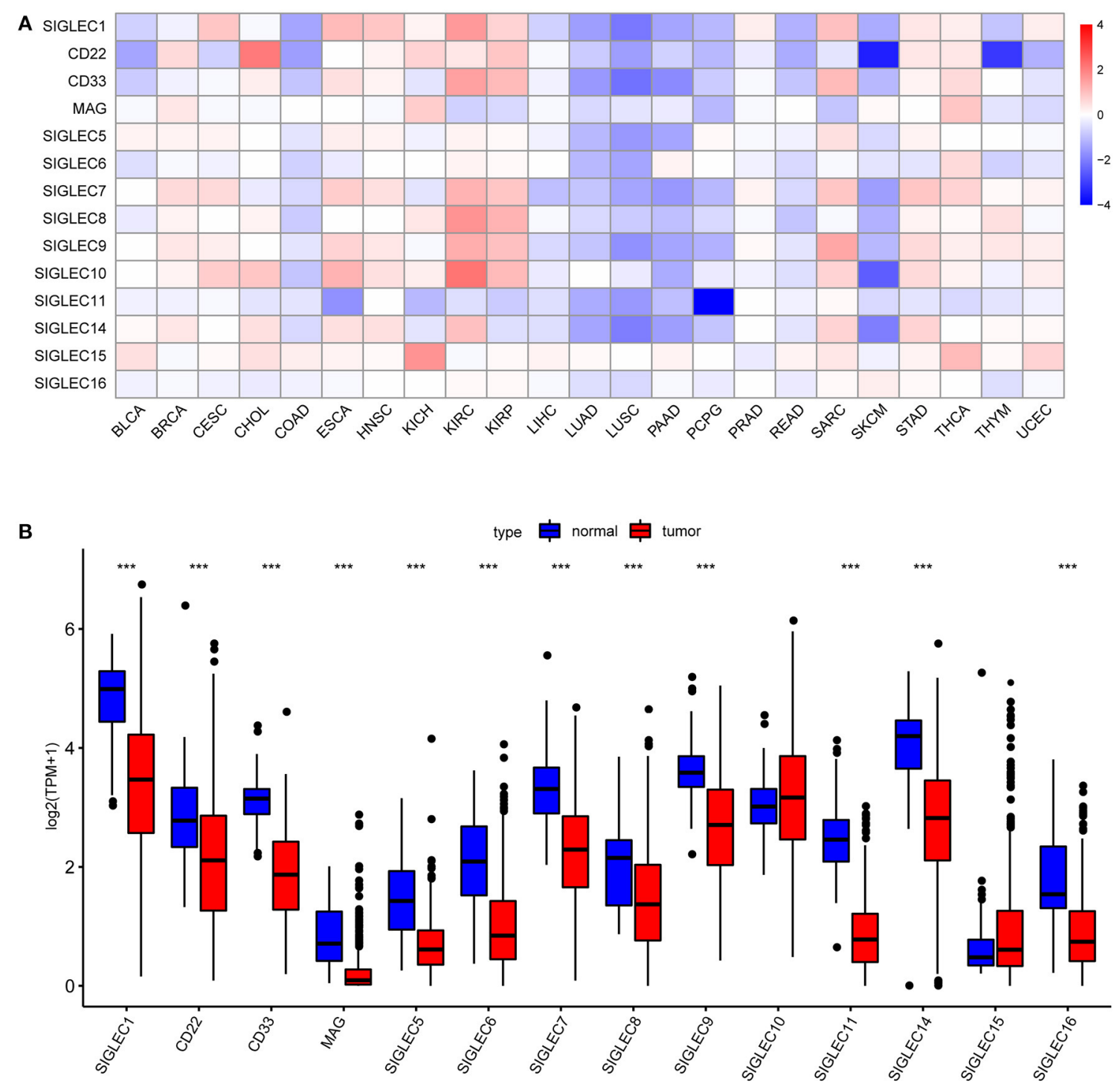

FIGURE 1 | SIGLEC expression in different tumor types. (A) Increased or decreased SIGLEC expression in 23 cancer types according to comparison with normal tissues from TCGA data. Filled color represents expression fold change using gradient color from blue to red. Blue represents down-regulation, and red represents up-regulation. (B) Boxplots showing S/GLEC expression following $\log _{2}(T P M+1)$ normalization of data for normal tissues and LUAD tissues using TCGA RNA-seq data ${ }^{* \star *} p<0.001$.

Analysis of genetic alteration frequency and types for the SIGLECs and according to tumor type (Figure 3B) showed that the alteration frequency ranged from 0.4 to $6 \%$, with $C D 22$ having the largest percentage change, followed by MAG. Additionally, amplifications occurred in $C D 22$ and $M A G$, with mutations were the most common features and included truncation, missense, and in-frame mutations. Moreover, we found deep deletions in all SIGLECs.

Using the LUAD dataset from TCGA $(n=586)$, we performed gene co-expression analysis to determine correlations between SIGLEC family members. The results showed significant positive correlations between SIGLEC1, CD33, SIGLEC5, SIGLEC7, SIGLEC9, SIGLEC11, and SIGLEC1 expression. Additionally, expression of SIGLEC10 was strongly positively correlated with that of SIGLEC5, SIGLEC7, SIGLEC8, SIGLEC9, and SIGLEC14 expression (Figure 3C), whereas no correlation between $M A G$ and other SIGLEC family members were observed, except for CD22 and SIGLEC6.

We then evaluated other genes co-expressed with SIGLECs, focusing on the top 30 genes of each SIGELC, with deletion of duplicates ( $n=198$ genes). Following screening of the 3,284 differentially expressed LUAD genes, we identified 105 overlapping genes related to SIGLEC expression and differentially expressed in LUAD (Figure 3D).

\section{PPI-Network Construction}

To assess potential relationship between the identified genes, we generated a PPI network. As shown in Figure 4A, SIGLEC6 and 


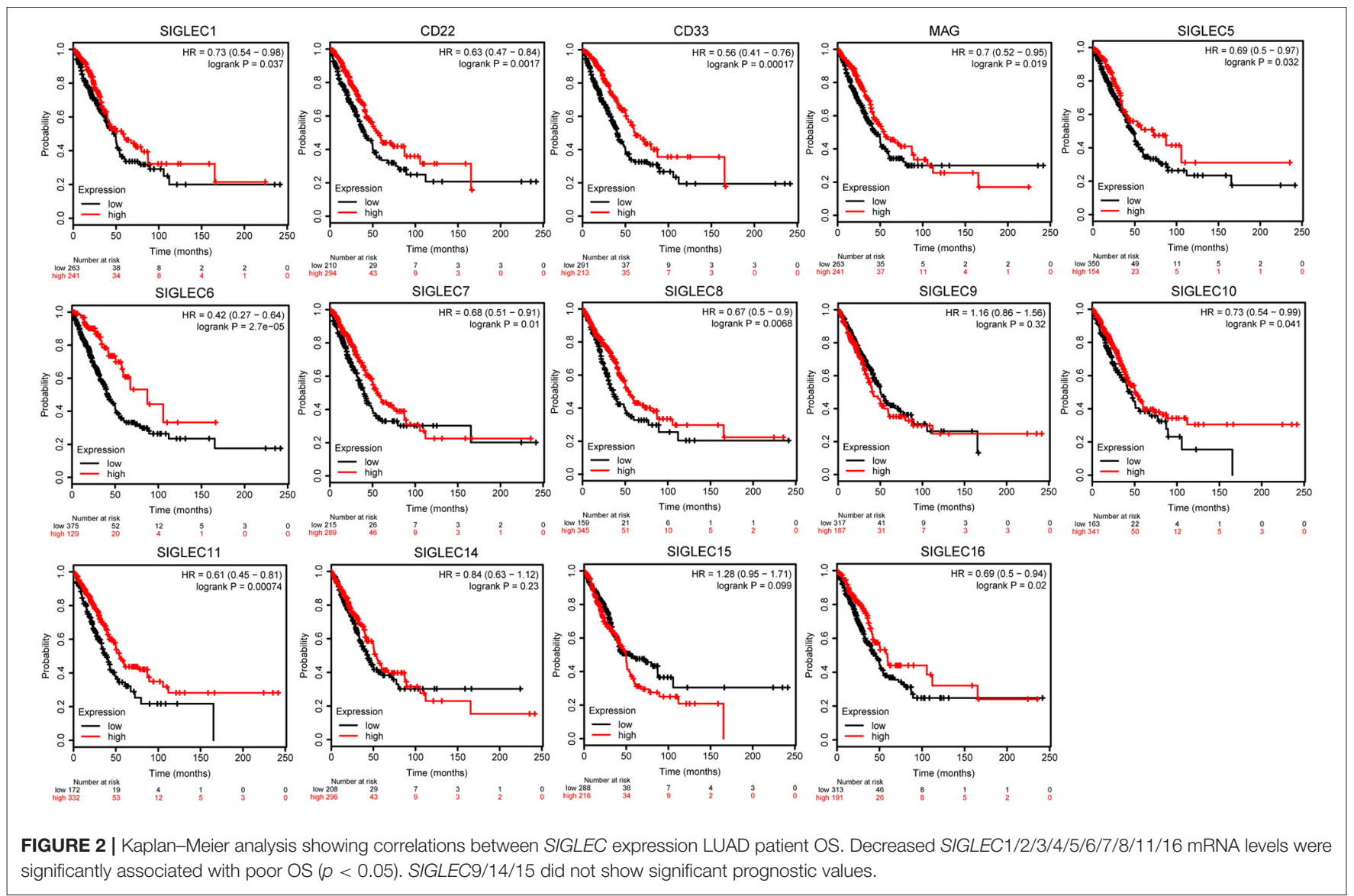

SIGLEC16 did not interact with other genes. We subsequently identified 10 hub genes (Figure 4B).

\section{GO and KEGG Analyses}

We then performed DAVID analysis of proteins in the PPI network for functional and pathway enrichment. We selected 20 GO terms (Figure 5A), which included cell adhesion, inflammatory response, cell-surface receptorsignaling pathway, B cell receptor-signaling pathway, signal transduction, cellular response to mechanical stimulus, positive regulation of tumor necrosis factor (TNF) production, and positive regulation of $\mathrm{T}$ cell proliferation among biological processes significantly associated with LUAD tumorigenesis and progression. Cellular component (CC) analysis indicated enrichment in integral components of the membrane, rough endoplasmic reticulum, extracellular exosome, and immunological synapse. Areas associated with molecular function (MF) were enriched for carbohydrate binding, receptor activity, transmembrane signaling-receptor activity, integrin binding, and non-membrane-spanning protein tyrosine kinase activity. Moreover, KEGG pathway analysis identified osteoclast differentiation, hematopoietic cell lineage, cell-adhesion molecules, the phagosome, primary immunodeficiency, Fc gamma R-mediated phagocytosis, complement and coagulation cascades, tuberculosis, the chemokine-signaling pathway, and asthma as enriched pathways (Figure 5B).

\section{SIGLECs Correlation With Immune-Cell Infiltration and -Molecule Levels, Respectively}

Given that the enrichment-analysis results indicated SIGLEC involvement with various immune-activation and -regulation processes, we evaluated correlations between the SIGLEC levels and immune-cell infiltration and immune-molecule levels. The results showed that levels of all SIGLEC family members were negatively correlated with tumor purity. SIGLEC1, CD22, SIGLEC3, SIGLEC5, SIGLEC6, SIGLEC7, SIGLEC8, SIGLEC9, SIGLEC10, SIGLEC11, and SIGLEC14 displayed similar immune-cell profiles, showing positive correlations with infiltrating levels of $\mathrm{B}$ cells, $\mathrm{CD}^{+} \mathrm{T}$ cells, $\mathrm{CD}^{+} \mathrm{T}$ cells, macrophages, neutrophils, and dendritic cells. Compared with their correlations with B cell, CD8+ $\mathrm{T}$ cell, and $\mathrm{CD} 4+\mathrm{T}$ cell infiltration $(r>0.3)$, SIGLEC1 (CD33), SIGLEC5, SIGLEC7, SIGLEC9, SIGLEC11, and SIGLEC14 showed a higher correlation with macrophage, neutrophil, and dendritic cell infiltration $(r>0.5)$. However, no correlation was found between MAG and these immune cells. Additionally, SIGLEC15 and SIGLEC16 were significantly correlated with neutrophil and dendritic cell infiltration 


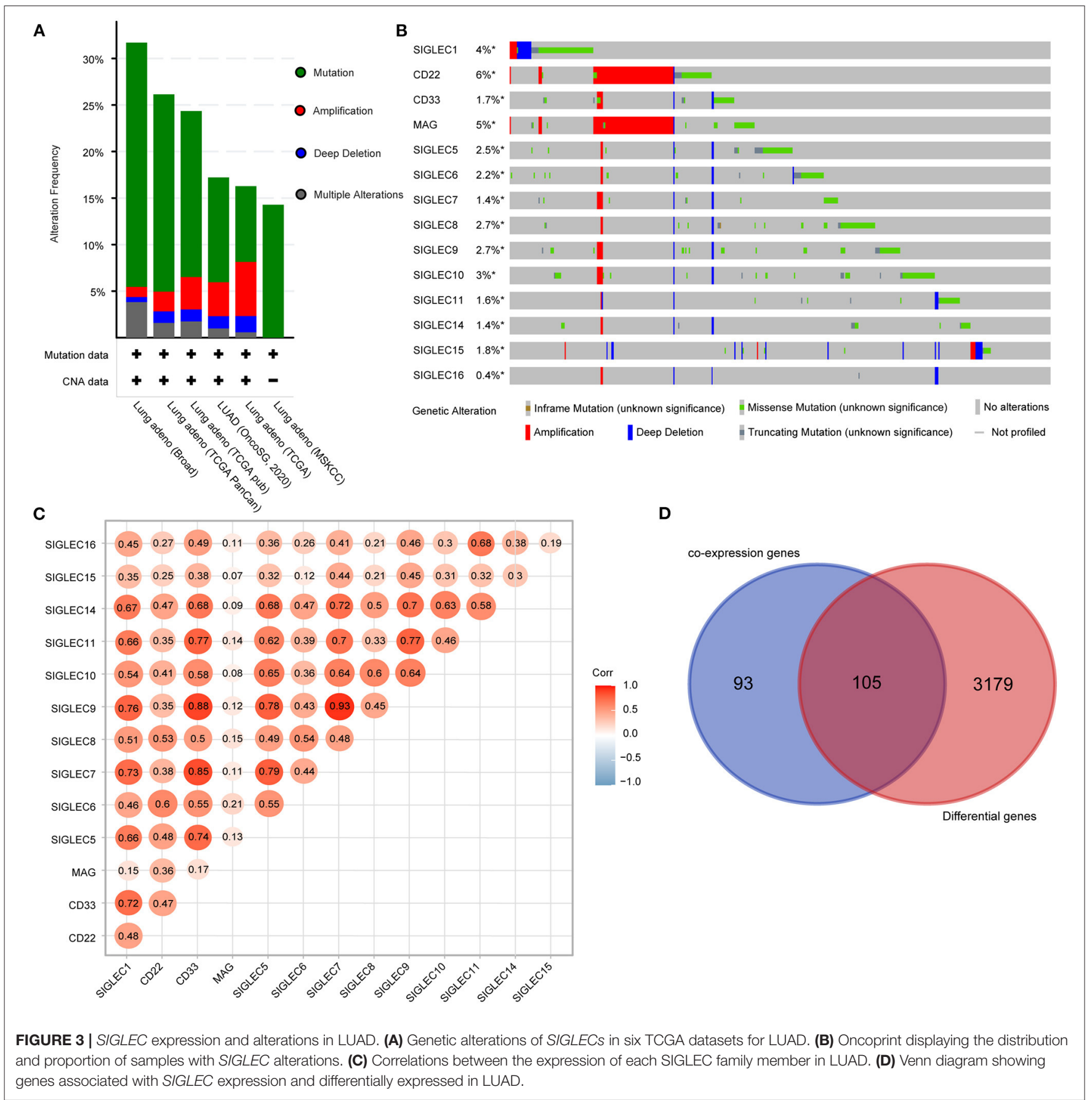

$(p<0.05)$, and a strong positive correlation was observed between $\mathrm{CD} 22$ and $\mathrm{B}$ cell and $\mathrm{CD}^{+} \mathrm{T}$ cell infiltration (Figure 6A).

To further investigate immune-related activity associated with SIGLECs in vivo, we analyzed correlations between SIGLEC family members with immunosuppressive molecules, such as adenosine $\mathrm{A} 2 \mathrm{a}$ receptor (ADORA2A), CD96, indoleamine 2,3-dioxygenase 1 (IDO1), CTLA4, programmed cell death 1 (PDCD1), lymphocyte-activation gene 3 (LAG3), $\mathrm{T}$ cell immunoreceptor with Ig and ITIM domains (TIGIT), transforming growth factor- $\beta$ receptor 1 (TGFBR1), and immunostimulatory molecules, such as CD27, CD276, CD48, CD70, killer cell lectin-like receptor C1, MHC class I polypeptide-related sequence $\mathrm{B}, \mathrm{IL}-6,5$ ' nucleotidase ecto (NT5E), poliovirus receptor precursor (PVR), glucocorticoidinduced TNF receptor (GITR), and OX40. In LUAD, the immunosuppressive molecules CD244, CD274, colony stimulating factor 1 receptor (CSF1R), hepatitis A virus cellular 

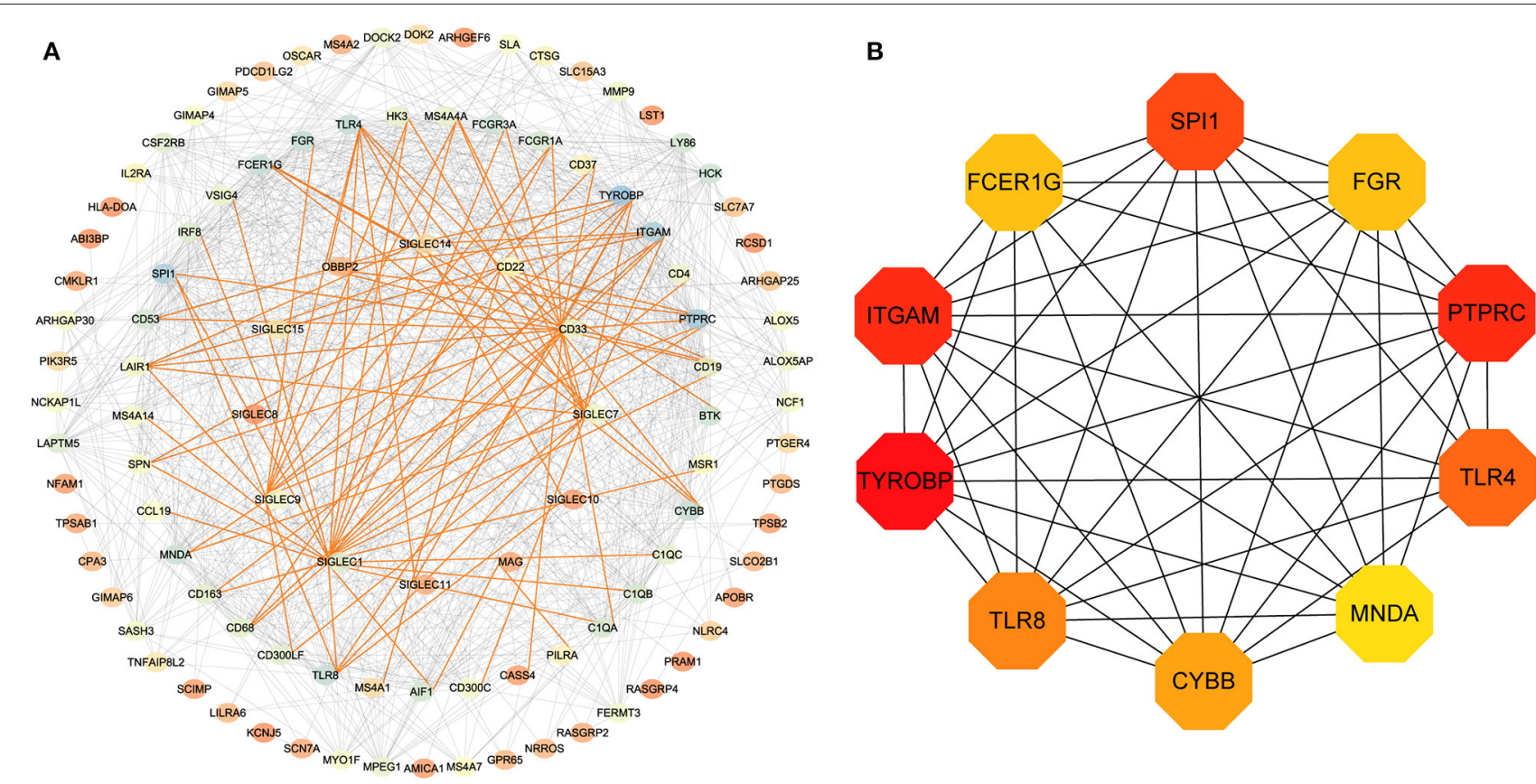

FIGURE 4 | PPI-network analysis. (A) Network of proteins encoded by differentially expressed genes in LUAD and related to SIGLEC expression. The gradient of the node color from red to blue represents increases in the number of edges. The red lines represent the direct interaction between SIGLECs and differential genes. (B) According to the connectivity of the network, 10 genes with the highest degrees of connectivity were selected as hub genes.

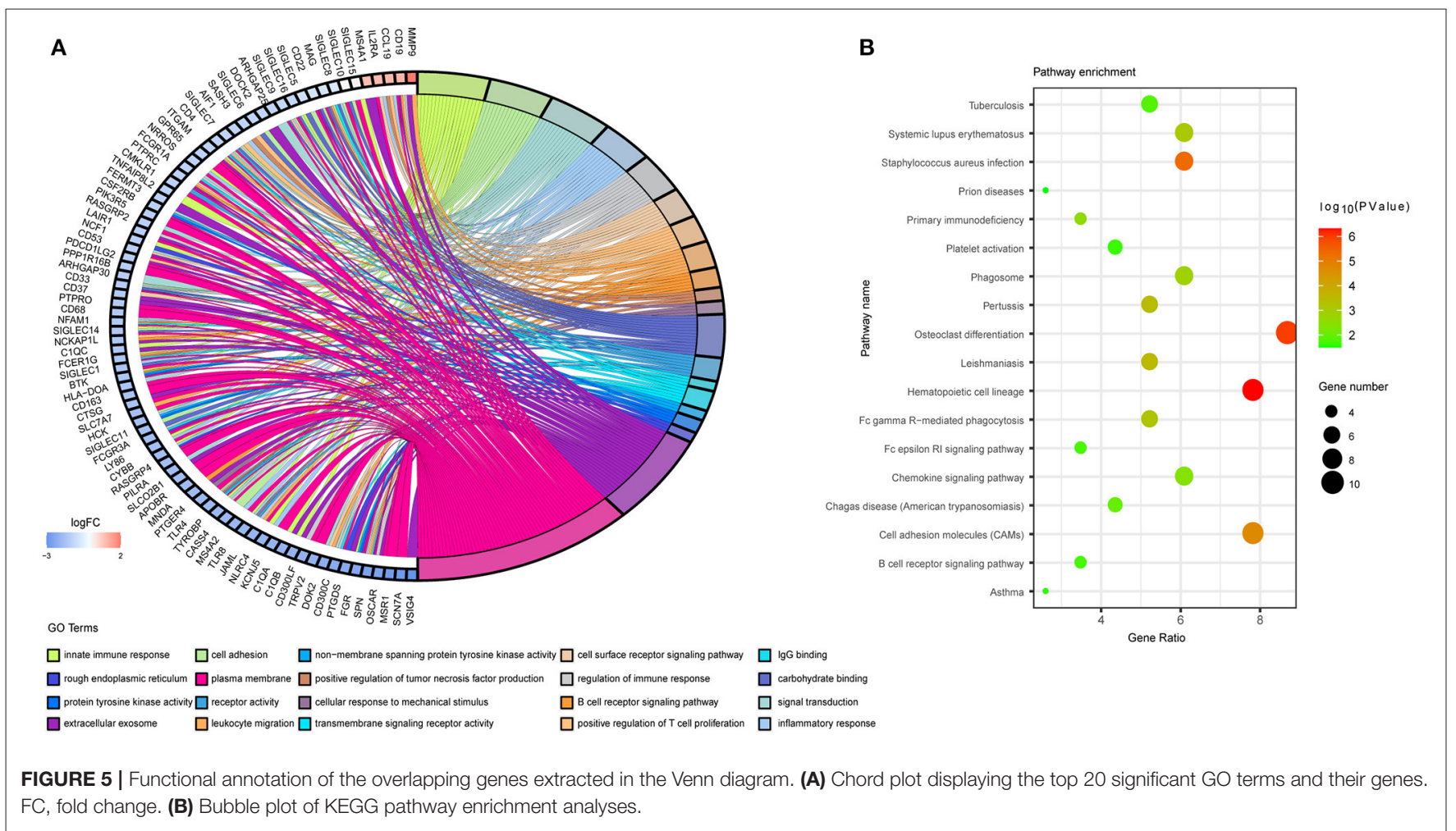

receptor 2 (HAVCR2), IL-10, PDCD1 ligand 2 (PDCD1LG2) showed a strong positive correlation with SIGLEC1, CD33, SIGLEC7, SIGLEC8, SIGLEC9, SIGLEC10, and SIGLEC14, and CD22 was strongly positively correlated with ADORA2A, B- and T-lymphocyte attenuator (BTLA), CD96, CTLA4, and TIGIT $(r>0.5)$. Additionally, SIGLEC11 was strongly 

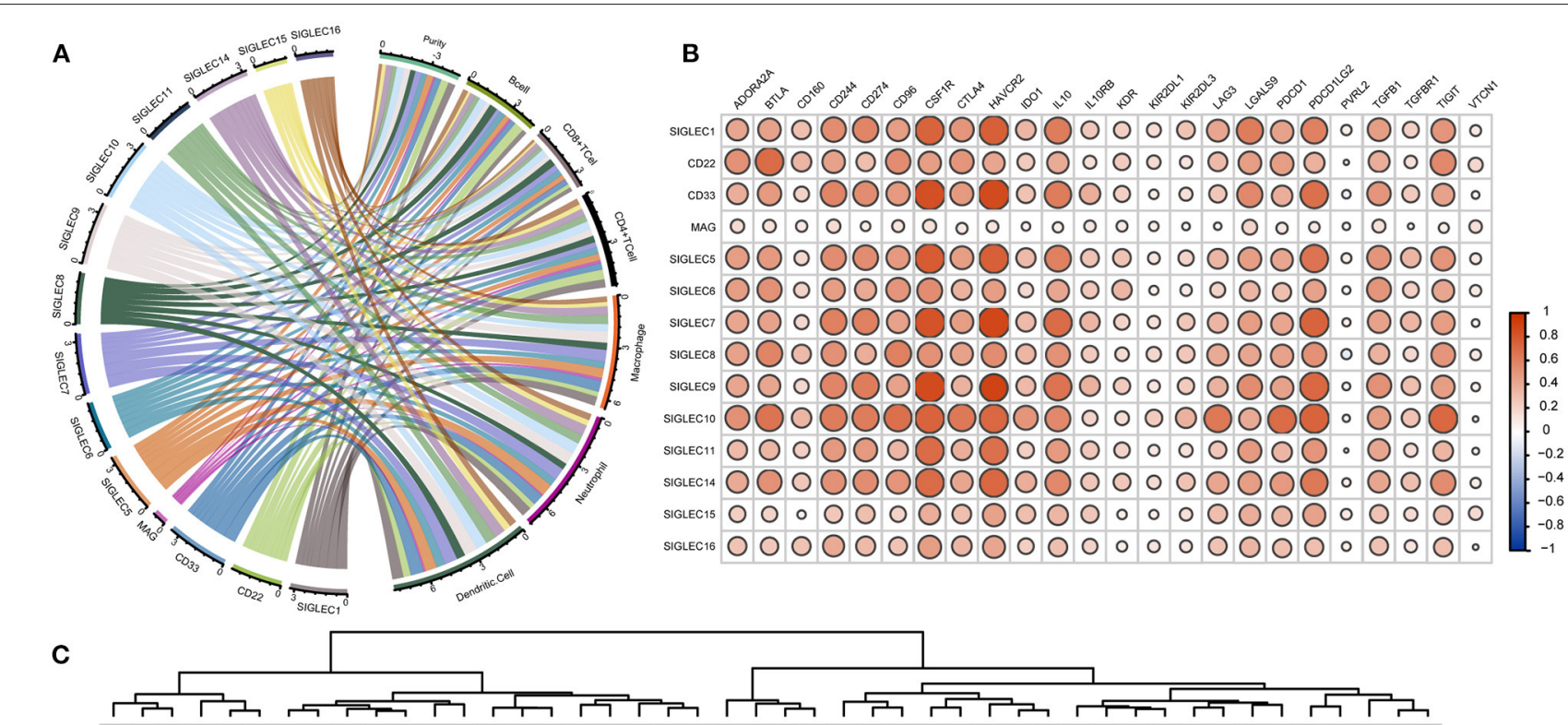

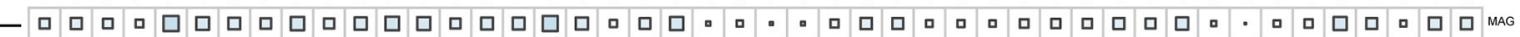

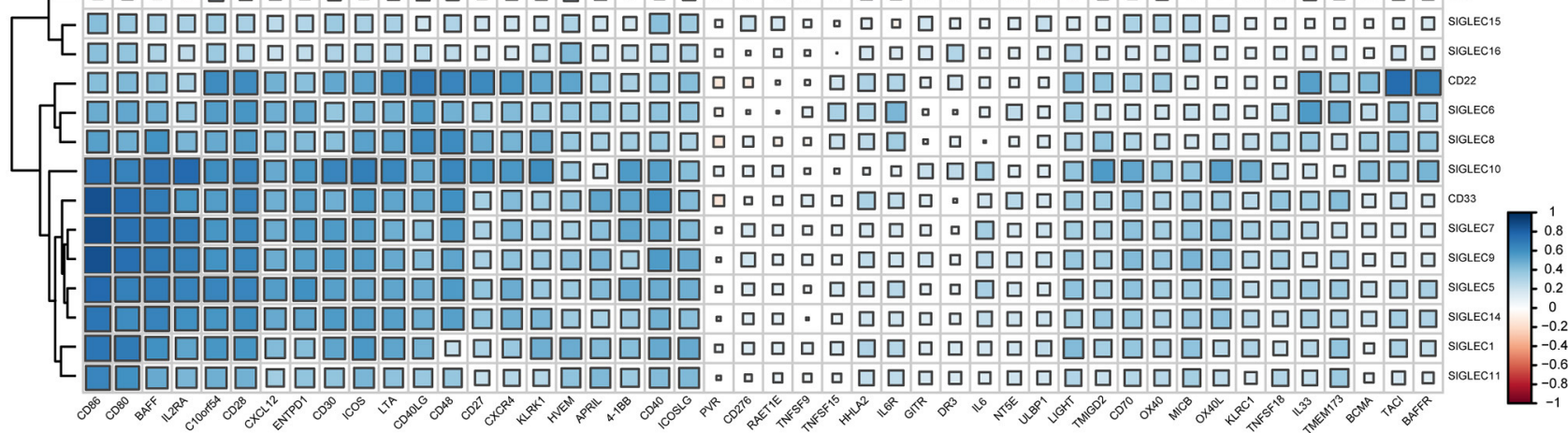

FIGURE 6 | SIGLECs expression is highly correlated with immune-cell infiltration and immune-molecule in LUAD. (A) Relationship between SIGLECs and immune-cell infiltration in LUAD. Line thickness and the size of the area corresponding to SIGLECs and immune cells represent the size of the Spearman's rho value. (B) Correlations between SIGLECs and immunosuppressive molecules. (C) Correlations between SIGLECs and immunstimulatory molecules. The color and size of the squares represent the $R$-value of Spearman's correlation. Correlation coefficients were classified as weak $(0.1 \leq r<0.3)$, moderate $(0.3 \leq r<0.5)$, or strong $(r \geq 0.5)$.

positively correlated with CSF1R and HAVCR2, and there was a significant strongly positive correlation between SIGLEC10 and ADORA2A, BTLA, CD96, IDO1, LAG3, PDCD1, PDCD1LG2, and TIGIT $(p<0.05, r>0.5)$. By contrast, SIGLEC family members showed a weak association with CD160, IL-10RB, kinase insert domain receptor, killer cell immunoglobulinlike receptor two Ig domains and long cytoplasmic tail (KIR2DL) 1, KIR2DL3, PVR ligand 2, TGFBR1, and V-set domain-containing $\mathrm{T}$ cell activation inhibitor 1 , whereas MAG had no association with any immunosuppressive molecule (Figure 6B).

Analysis of associations with immune-stimulating molecules revealed no correlation between SIGLECs and CD276, HERVH LTR-associating 2, IL-6, NT5E, PVR, retinoic acid early transcript 1E, GITR, death receptor 3, TNF superfamily member (TNFSF)15, TNFSF9, UL16-binding protein 1 (ULBP1) (Figure 6C). However, a moderate-to-strong positive correlation was found between $\mathrm{V}$-domain Ig suppressor of $\mathrm{T}$ cell activation (VISTA), CD28, CD40, CD80, CD86, C-X-C chemokine (CXC) ligand 12, CXC receptor 4, ectonucleoside triphosphate diphosphohydrolase 1 , inducible $\mathrm{T}$ cell co-stimulator (ICOS), IL2 receptor- $\alpha$, ICOS ligand, lymphotoxin $\alpha, \mathrm{CD} 30,4-1 \mathrm{BB}$, and $\mathrm{B}$ cell-activating factor (BAFF) with $\mathrm{CD} 22, \mathrm{CD} 33$, and SIGLEC1/5/6/7/8/9/10/11/14. IL33 was strongly correlated with CD22 and SIGLEC6. Notably, that there was also a strong correlation between $\mathrm{CD} 22$ and $\mathrm{CD} 27$, transmembrane activator and CAML interactor, BAFF receptor.

\section{DISCUSSION}

SIGLEC family members play significant and diverse roles in autoimmune diseases and tumor progression $(42,43)$, making SIGLECs and sialoglycans attractive targets for anticancer immunotherapies (44). Compared with healthy cells, cancer 
cells sport a higher density of glycan structures terminating in sialic acid, the ligand for SIGLECs. Tumor cells can engage the sialoglycan-Siglec axis to evade immune control. Interference with sialoglycan synthesis or sialoglycan-Siglec interactions might enhance antitumor immunity $(32,45)$. In the present study, we analyzed SIGLEC expression in multiple tumors relative to normal tissues and comprehensively analyzed relationships between their differential expression and OS in LUAD patients. The results were consistent with previous reports, specifically that SIGLEC15 is upregulated in various tumors, including bladder urothelial carcinoma, cervical squamous cell carcinoma and endocervical adenocarcinoma, CHOL, colon adenocarcinoma, LUAD, KICH, KIRP, thyroid carcinoma, and uterine corpus endometrial carcinoma to varying degrees and can be used as a novel immune checkpoint for tumor immunotherapy (46). One study performed immunohistochemistry to evaluate the expression and prognostic value of SIGLEC15 in a cohort of 103 LUAD specimens. The results showed that patients with positive SIGLEC15 expression had an unfavorable progression free Survival. There was no significant difference in OS between SIGLEC15 positive and negative patients. In TCGA data, it was found that upregulated SIGLEC15 expression was associated with longer OS in breast invasive carcinoma, BLCA, THCA, head and neck squamous cell carcinoma and UCEC and with longer relapse-free survival (RFS) in liver hepatocellular carcinoma, ovarian serous cystadenocarcinoma, BRCA, and UCEC. By contrast, up-regulated SIGLEC15 expression was associated with shorter OS in kidney renal clear cell carcinoma, pancreatic adenocarcinoma and Sarcoma, and with shorter RFS in SARC and PAAD (47). In a recent article, researchers showed for the first time the in situ expression of SIGLEC6 by mast cell (MC) in human colorectal cancer (CRC) tissues. SIGLEC6 is up-regulated on MC when stimulated with hypoxia or colon cancer cells. However, SIGLEC6 expression was not detected in colon cell lines. These findings supported that SIGLEC6 can modulate MC activity in the CRC tumor microenvironment (48). As an inhibitory receptor, SIGLEC6 plays a major role in both the proliferation and effector functions of tissue-like memory B cells (49). SIGLEC6 is expressed on plasmacytoid dendritic cell leukemia, but not on cutaneous granulocytic monocytic leukemia (50). In addition, compared with normal lymphoid cells and some other lymphoid malignancies, SIGLEC6 is highly expressed in mucosa-associated lymphoid tissue lymphoma (51). Tuscano (52) previously characterized the expression of CD22 in lung cancer cells and patient samples, identifying CD22 as a target for therapeutic intervention in lung cancer. However, recent studies repeating this experiment found that CD22 was not expressed at measurable levels on the surface of lung cancer cells, and that tumor cells could not be killed by anti-CD22 immunotoxins (53). In the present study, data based on TCGA database, we showed that CD22 was downregulated in LUAD tissue relative to normal tissue, with the same phenomenon reported in lung squamous cell carcinoma.

Downregulation of SIGLEC1, CD22, CD33, MAG, SIGLEC5, SIGLEC6, SIGLEC7, SIGLEC8, SIGLEC11, and SIGLEC16 in LUAD was not only associated with poor OS but also related to the immune response and cell adhesion according to GO and KEGG analyses. As is known, the immune function of the host is closely related to tumor progression. Immune evasion by cancer cells can promote tumor progression. Cell-adhesion molecules play an important role in tumor invasion and metastasis, both of which are related to changes in adhesion-molecule expression (54). Reduced expression of certain adhesion molecules in tumor cells can weaken adhesion between tumor cells, resulting in their separation from the primary tumor body. Additionally, certain adhesion molecules expressed on tumor cells in blood circulation to adhere to vascular endothelial cells and basement membranes. In the present study, our data indicated that SIGLECs might play roles in LUAD occurrence and development.

Gene mutation is an important factor in biological evolution and cancer. Previous studies show that an R122C substitution in human SIGLEC12 results in an inability of proteins to bind sialic acids (55). Additionally, the SIGLEC10 expressed by tumor-associated macrophages can interact with CD24 to promote immune evasion (56). Additionally, SIGLEC14 expressed in a monocytic cell line interacts with DAP12 to enhance lipopolysaccharide-induced TNF- $\alpha$ secretion, and gene fusion SIGLEC5 and SIGLEC14 results in functional deletion of SIGLEC14 (57). The loss of SIGLEC14 caused by SIGLEC14null allele homozygosity is related to a reduced risk of chronic obstructive pulmonary disease exacerbation (58). CD33 single nucleotide polymorphisms (SNPs) have been implicated in the risk of Alzheimer's disease $(\mathrm{AD})$ and the therapeutic efficacy of acute myeloid leukemia (AML) (59). The $\mathrm{C}>\mathrm{T}$ allele of rs12459419 leads to an Ala (codon GCC) to Val (codon GTC) amino acid change. The Ala14Val change is present in the signal peptide (amino acids 1-17), which is likely to lead to a decrease in CD33 expression and thus affect the dose and efficacy of GO therapy in patients with AML $(60,61)$. The CD33 polymorphism rs3865444 and its functional proxy rs124549419 are associated with exon 2 splicing efficiency. The shorter CD33-isoform (D2-CD33), generated as a result of alternate splicing, lacking the ligand binding domain represents a gain of function variant that reduces Alzheimer's disease risk (62). In the present study, we found that SIGLEC mutations were the most common alteration in LUAD. Whether these mutations will change the protein structure and cause corresponding functional enhancement or impairment of function remains to be elucidated.

Immune-cell infiltration into tumor tissues, as well as their specific type, distribution, and tissue localization, is of particular importance for tumor development and prognosis (63). In the LUAD TME, tumor-infiltrating immune cells are double-edged sword in the development of lung cancer. On the one hand, they attack and kill cancer cells to inhibit tumor progression; however, they also screen tumor cells that are better-suited for survival in an immunoreactive host, change the TME, or are assimilated by lung cancer cells, ultimately promoting tumor progression (64). Recent studies suggest that CD169+ macrophages in tumor regional lymph nodes are positively associated with favorable prognosis in patients with colorectal cancer, bladder cancer, endometrial carcinoma, or malignant melanoma (65-68). SIGLECs show distinct expression patterns 
on different cell types. Human SIGLECs are predominantly expressed on the surface of immune cells, and most immune cells of the tumor microenvironment are co-regulated by the action of SIGLECs. SIGLEC1 is mainly expressed on macrophages and dendritic cells. CD22 is expressed on B cells, conventional dendritic cells and mast cells. CD33 has a more diverse expression as $\mathrm{CD} 33$ is present on myeloid progenitors, monocytes, macrophages, microglia and granulocytes. MAG is expressed only on oligodendrocytes and schwann cells. SIGLEC5 is myeloid-specific, but unlike CD33, it is expressed on neutrophils, monocytes, B cells and mast cells. SIGLEC6, a leptin-binding modulator, is expressed on B cells, monocytes and placental trophoblasts. SIGLEC7 is expressed on natural killer cells, monocytes, mast cells and T cells. SIGLEC8 is found only on "allergic cells" (basophils, mast cells, eosinophils), while SIGLEC9 is expressed on neutrophils, monocytes, natural killer cells, conventional dendritic cells and T cells. SIGLEC10 is present on some B cells, monocytes and eosinophils. SIGLEC11 appears to be restricted to macrophages and microglia. SIGLEC14 is expressed on neutrophils and monocytes, and SIGLEC15 is expressed on osteoclasts, macrophages and dendritic cells. SIGLEC16 is present on macrophages and microglia $(19,26$, 69). In the present study, we found that $\mathrm{CD} 22, \mathrm{CD} 33$, and SIGLEC1/5/6/7/8/9/10/11/14/15/16 were significantly negatively associated with tumor purity and significantly positively associated with $\mathrm{B}$ cell, $\mathrm{CD} 8^{+} \mathrm{T}$ cells, and $\mathrm{CD} 4^{+} \mathrm{T}$ cell infiltration and moderately $(r>0.3)$-to-strongly $(r>0.5)$ correlated with neutrophil, macrophage, and dendritic cell infiltration. Dendritic cells are antigen-presenting cells (APCs) and central to initiating, regulating, and maintaining immune responses while also playing an important role in inducing antitumor immune responses (70). Dendritic cell-based tumor vaccines have been tested clinically and achieved positive outcomes $(71,72)$.

Levels of immune molecules in the TME are closely related to patient receipt of immunotherapy and reactivity following treatment (73). In the present study, we showed correlations between SIGLEC family members and LUADrelated levels of immune molecules, including the immunestimulatory checkpoint molecules CD27, CD28, OX40, ICOS and the immune-inhibitory checkpoint molecules CTLA4, PD1, ADORA2A, BTLA, LAG3, and VISTA. CD28 is expressed on the surface of $\mathrm{T}$ cells and required for co-stimulatory signaling essential for $\mathrm{T}$ cell activation, proliferation, and survival, as well as T helper 2 cell development. CD28 binds CD80 and CD86 on the surface of APCs to initiate co-stimulatory signaling to T cells (74). By contrast, CTLA-4 delivers a co-inhibitory signal via CD80/CD86 (75). A recent study reported that SIGLEC9expressing $\mathrm{T}$ cells co-expressed several inhibitory receptors, including $\mathrm{T}$ cell Ig- and mucin-domain-containing protein 3, PD1, and LAG3 (25). ICOS expressed on the surface of activated $\mathrm{T}$ cells can enhance all basic $\mathrm{T}$ cell responses to a foreign antigen according to binding of its unique ligand is ICOSL, which initiates a pathway that enhances antitumor immune responses. Combining ICOS-agonistic or -antagonistic antibodies with CTLA-4 or PD-1/PD-L1 might produce potent synergistic effects
(76). BTLA, an immunomodulatory receptor similar to CTLA4 and PD-1, binds to herpesvirus-entry mediator to initiate co-suppressive signals and play a negative regulatory role in the antitumor immune response. This molecule is related to tumor immune evasion and might represent a potential target for tumor immunotherapy (77). Despite therapies targeting CTLA4, PD1, and PDL1 have shown success in many cancers, although not all patients respond well to these therapies. TIGIT and CD96 are expressed on the surface of $\mathrm{T}$ cells and natural killer cells and represent targets for immune modulation (78), as blocking CD96 or TIGIT with monoclonal antibodies improves tumor control in mice, especially when used in combination with PD-1/PD-L1 blockade (79). IL-33, an activator of T cells, mediates its immune response mainly through polarized $\mathrm{T}$ helper 2 cells. A study showed that IL-33 enhances SIGLEC8induced eosinophil apoptosis (80). Overexpression of CSF1R or its ligand CSF1 is found in various solid tumors, including those associated with breast, prostate, and ovarian cancer, and plays an important role in tumor malignancy and metastasis (81). Moreover, the CSF1/CSF1R pathway is a dominant regulator of macrophage differentiation and function, with studies showing that CSF1R inhibition can deplete tumor associated macrophages and improve $\mathrm{T}$ cell responses $(82,83)$. In the present study, correlations between SIGLECs and immune molecules suggest an underlying novel feature of SIGLEC-driven immune response and regulation of immune infiltrating cells. Remarkably, we found that MAG has no or only a very weak correlation with these immune cells and immune molecules. This result seems reasonable, since MAG, in contrast to other SIGLECs, appears to be solely expressed in myelin oligodendrocytes and Schwann cells but not in human immune cells and does not carry any immunoreceptor tyrosine-based inhibitory motifs (84).

This study had limitations. First, the analyses were conducted using publically available databases, and the sample size of the data was relatively small. Second, the results lack experimental and clinical validation. Third, the precise mechanism by which SIGLECs function in LUAD requires further study. LUAD tumorigenesis results from abnormal expression of many genes. Therefore, we hope that these data promote further exploration of targets affecting LUAD occurrence and development.

In conclusion, this study showed that downregulated expression of SIGLEC1, CD22, CD33, MAG, SIGLEC5, SIGLEC6, SIGLEC7, SIGLEC8, SIGLEC11, SIGLEC14, and SIGLEC16 in LUAD was associated with poor prognosis. Additionally, we found that SIGLEC mutations in LUAD account for a high proportion of genetic alterations. Moreover, we demonstrated various correlations between SIGLEC levels and different immune molecules, as well as levels of immune-cell infiltration of the TME, offering novel insights and enhancing our understanding of the potential relationships between the TME and LUAD prognosis. Furthermore, these findings might help explain why abnormal expression of certain SIGLEC family members is related to poor prognosis in LUAD patients and thereby offer a useful reference for personalized immunotherapy and predicting the efficacy of immune-checkpoint blockade. 


\section{DATA AVAILABILITY STATEMENT}

The datasets presented in this study can be found in online repositories. The names of the repository/repositories and accession number(s) can be found in the article/supplementary material.

\section{AUTHOR CONTRIBUTIONS}

QY, SN, and HZ developed the study concept and designed the research. HZ analyzed the data and drafted the manuscript. YX,

\section{REFERENCES}

1. Bray F, Ferlay J, Soerjomataram I, Siegel R, Torre L, Jemal A. Global cancer statistics 2018: GLOBOCAN estimates of incidence and mortality worldwide for 36 cancers in 185 countries. CA Cancer J Clin. (2018) 68:394424. doi: 10.3322/caac.21492

2. Molina J, Yang P, Cassivi S, Schild S, Adjei A. Non-small cell lung cancer: epidemiology, risk factors, treatment, and survivorship. Mayo Clin Proc. (2008) 83:584-94. doi: 10.4065/83.5.584

3. Sun S, Schiller J, Gazdar A. Lung cancer in never smokers-a different disease. Nat Rev Cancer. (2007) 7:778-90. doi: 10.1038/nrc2190

4. Kris M, Johnson B, Berry L, Kwiatkowski D, Iafrate A, Wistuba I, et al. Using multiplexed assays of oncogenic drivers in lung cancers to select targeted drugs. JAMA. (2014) 311:1998-2006. doi: 10.1001/jama.2014.3741

5. Dogan S, Shen R, Ang D, Johnson M, D'Angelo S, Paik P, et al. Molecular epidemiology of EGFR and KRAS mutations in 3,026 lung adenocarcinomas: higher susceptibility of women to smoking-related KRAS-mutant cancers. Clin Cancer Res. (2012) 18:6169-77. doi: 10.1158/1078-0432.Ccr-11-3265

6. Roviello G. The distinctive nature of adenocarcinoma of the lung. Oncotargets Ther. (2015) 8:2399-406. doi: 10.2147/ott.S89225

7. Pan Y, Zhang Y, Li Y, Hu H, Wang L, Li H, et al. ALK, ROS1 and RET fusions in 1139 lung adenocarcinomas: a comprehensive study of common and fusion pattern-specific clinicopathologic, histologic and cytologic features. Lung Cancer. (2014) 84:121-6. doi: 10.1016/j.lungcan.2014.02.007

8. Drilon A, Wang L, Hasanovic A, Suehara Y, Lipson D, Stephens $\mathrm{P}$, et al. Response to cabozantinib in patients with RET fusionpositive lung adenocarcinomas. Cancer Discov. (2013) 3:6305. doi: 10.1158/2159-8290.Cd-13-0035

9. Cancer Genome Atlas Research Network. Comprehensive molecular profiling of lung adenocarcinoma. Nature. (2014) 511:543-50. doi: 10.1038/nature13385

10. Herbst R, Morgensztern D, Boshoff C. The biology and management of nonsmall cell lung cancer. Nature. (2018) 553:446-54. doi: 10.1038/nature25183

11. Anagnostou V, Brahmer J. Cancer immunotherapy: a future paradigm shift in the treatment of non-small cell lung cancer. Clin Cancer Res. (2015) 21:976-84. doi: 10.1158/1078-0432.Ccr-14-1187

12. Leach D, Krummel M, Allison J. Enhancement of antitumor immunity by CTLA-4 blockade. Science. (1996) 271:17346. doi: $10.1126 /$ science.271.5256.1734

13. Hodi F, O'Day S, McDermott D, Weber R, Sosman J, Haanen J, et al. Improved survival with ipilimumab in patients with metastatic melanoma. $N$ Engl J Med. (2010) 363:711-23. doi: 10.1056/NEJMoa1003466

14. Dong H, Strome S, Salomao D, Tamura H, Hirano F, Flies D, et al. Tumorassociated B7-H1 promotes T-cell apoptosis: a potential mechanism of immune evasion. Nat Med. (2002) 8:793-800. doi: 10.1038/nm730

15. Iwai $\mathrm{Y}$, Ishida $\mathrm{M}$, Tanaka $\mathrm{Y}$, Okazaki $\mathrm{T}$, Honjo $\mathrm{T}$, Minato N. Involvement of PD-L1 on tumor cells in the escape from host immune system and tumor immunotherapy by PD-L1 blockade. Proc Natl Acad Sci USA. (2002) 99:12293-7. doi: 10.1073/pnas.192461099

16. Hirsch FR, Scagliotti GV, Mulshine JL, Kwon R, Curran WJ, Jr., et al. Lung cancer: current therapies and new targeted treatments. Lancet. (2017) 389:299-311. doi: 10.1016/S0140-6736(16)30958-8
HY, XX, ZH, YY, and WX revised the manuscript. All authors read and approved the final version.

\section{FUNDING}

This work was supported by a joint project of the Luzhou Municipal Government and Southwest Medical University (2019LZXNYDC04, 2018LZXNYD-ZK26, and 2018LZXNYD-ZK38), and by a joint project of the People's Government of Luxian County and Southwest Medical University (2019LXXNYKD-06).

17. Sharma P, Hu-Lieskovan S, Wargo JA, Ribas A. Primary, adaptive, and acquired resistance to cancer immunotherapy. Cell. (2017) 168:70723. doi: 10.1016/j.cell.2017.01.017

18. O'Reilly M, Paulson J. Siglecs as targets for therapy in immunecell-mediated disease. Trends Pharmacol. Sci. (2009) 30:2408. doi: 10.1016/j.tips.2009.02.005

19. Crocker P, Paulson J, Varki A. Siglecs and their roles in the immune system. Nat Rev Immunol. (2007) 7:255-66. doi: 10.1038/nri2056

20. Padler-Karavani V, Hurtado-Ziola N, Chang Y, Sonnenburg J, Ronaghy A, $\mathrm{Yu} \mathrm{H}$, et al. Rapid evolution of binding specificities and expression patterns of inhibitory CD33-related Siglecs in primates. FASEB J. (2014) 28:128093. doi: 10.1096/fj.13-241497

21. Macauley MS, Crocker PR, Paulson JC. Siglec-mediated regulation of immune cell function in disease. Nat Rev Immunol. (2014) 14:65366. doi: 10.1038/nri3737

22. Crocker P, Redelinghuys P. Siglecs as positive and negative regulators of the immune system. Biochem Soc Trans. (2008) 36:1467-71. doi: 10.1042/bst0361467

23. Rumer K, Post M, Larivee R, Zink M, Uyenishi J, Kramer A, et al. Siglec-6 is expressed in gestational trophoblastic disease and affects proliferation, apoptosis and invasion. Endocr Relat Cancer. (2012) 19:82740. doi: 10.1530/erc-11-0379

24. Malhotra S, Castilló J, Bustamante M, Vidal-Jordana A, Castro Z, Montalban X, et al. SIGLEC1 and SIGLEC7 expression in circulating monocytes of patients with multiple sclerosis. Mult Scler. (2013) 19:52431. doi: $10.1177 / 1352458512458718$

25. Stanczak $M$, Siddiqui $S$, Trefny $M$, Thommen $D$, Boligan $K$, von Gunten S, et al. Self-associated molecular patterns mediate cancer immune evasion by engaging Siglecs on T cells. J Clin Invest. (2018) 128:491223. doi: $10.1172 /$ jci120612

26. Duan S, Paulson J. Siglecs as immune cell checkpoints in disease. Annu Rev Immunol. (2020) 38:365-95. doi: 10.1146/annurev-immunol-102419-035900

27. Bornhöfft K, Goldammer T, Rebl A, Galuska S. Siglecs: a journey through the evolution of sialic acid-binding immunoglobulin-type lectins. Dev Comp Immunol. (2018) 86:219-31. doi: 10.1016/j.dci.2018.05.008

28. O'Hear C, Heiber J, Schubert I, Fey G, Geiger T. Anti-CD33 chimeric antigen receptor targeting of acute myeloid leukemia. Haematologica. (2015) 100:33644. doi: 10.3324/haematol.2014.112748

29. Sullivan-Chang L, O'Donnell R, Tuscano J. Targeting CD22 in B-cell malignancies: current status and clinical outlook. BioDrugs. (2013) 27:293304. doi: 10.1007/s40259-013-0016-7

30. Leonard J, Goldenberg D. Preclinical and clinical evaluation of epratuzumab (anti-CD22 IgG) in B-cell malignancies. Oncogene. (2007) 26:370413. doi: $10.1038 /$ sj.onc. 1210370

31. Pillai S, Netravali I, Cariappa A, Mattoo H. Siglecs and immune regulation. Annu Rev Immunol. (2012) 30:357-92. doi: 10.1146/annurev-immunol-020711-07 5018

32. van de Wall S, Santegoets $K$, van Houtum E, Büll C, Adema G. Sialoglycans and Siglecs can shape the tumor immune microenvironment. Trends Immunol. (2020) 41:274-85. doi: 10.1016/j.it.2020.0 2.001 
33. Tang Z, Li C, Kang B, Gao G, Li C, Zhang Z. GEPIA: a web server for cancer and normal gene expression profiling and interactive analyses. Nucleic Acids Res. (2017) 45:W98-102. doi: 10.1093/nar/gkx247

34. Kent W, Sugnet C, Furey T, Roskin K, Pringle T, Zahler A, et al. The human genome browser at UCSC. Genome Res. (2002) 12:9961006. doi: 10.1101/gr.229102

35. Ritchie M, Phipson B, Wu D, Hu Y, Law C, Shi W, et al. limma powers differential expression analyses for RNA-sequencing and microarray studies. Nucleic Acids Res. (2015) 43:e47. doi: 10.1093/nar/gkv007

36. Cerami E, Gao J, Dogrusoz U, Gross B, Sumer S, Aksoy B, et al. The cBio cancer genomics portal: an open platform for exploring multidimensional cancer genomics data. Cancer Discov. (2012) 2:4014. doi: 10.1158/2159-8290.Cd-12-0095

37. Gao J, Aksoy B, Dogrusoz U, Dresdner G, Gross B, Sumer S, et al. Integrative analysis of complex cancer genomics and clinical profiles using the cBioPortal. Sci Signal. (2013) 6:pl1. doi: 10.1126/scisignal.2004088

38. Szklarczyk D, Franceschini A, Wyder S, Forslund K, Heller D, Huerta-Cepas J, et al. STRING v10: protein-protein interaction networks, integrated over the tree of life. Nucleic Acids Res. (2015) 43:D447-52. doi: 10.1093/nar/gku1003

39. Shannon P, Markiel A, Ozier O, Baliga N, Wang J, Ramage D, et al. Cytoscape: a software environment for integrated models of biomolecular interaction networks. Genome Res. (2003) 13:2498-504. doi: 10.1101/gr.1239303

40. Huang dW, Sherman B, Lempicki R. Systematic and integrative analysis of large gene lists using DAVID bioinformatics resources. Nat Protoc. (2009) 4:44-57. doi: 10.1038/nprot.2008.211

41. Li T, Fan J, Wang B, Traugh N, Chen Q, Liu J, et al. TIMER: a web server for comprehensive analysis of tumor-infiltrating immune cells. Cancer Res. (2017) 77:e108-10. doi: 10.1158/0008-5472.Can-17-0307

42. Fraschilla I, Pillai S. Viewing Siglecs through the lens of tumor immunology. Immunol Rev. (2017) 276:178-91. doi: 10.1111/imr.12526

43. Lin C, Yeh Y, Yang K. Functions and therapeutic targets of Siglec-mediated infections, inflammations and cancers. J Formos Med Assoc. (2019) 120:524. doi: 10.1016/j.jfma.2019.10.019

44. Adams O, Stanczak M, von Gunten S, Läubli H. Targeting sialic acid-Siglec interactions to reverse immune suppression in cancer. Glycobiology. (2018) 28:640-7. doi: 10.1093/glycob/cwx108

45. Daly J, Carlsten M, O’Dwyer M. Sugar free: novel immunotherapeutic approaches targeting Siglecs and sialic acids to enhance natural killer cell cytotoxicity against cancer. Front Immunol. (2019) 10:1047. doi: 10.3389/fimmu.2019.01047

46. Wang J, Sun J, Liu L, Flies D, Nie X, Toki M, et al. Siglec-15 as an immune suppressor and potential target for normalization cancer immunotherapy. Nat Med. (2019) 25:656-66. doi: 10.1038/s41591-019-0374-x

47. Li B, Zhang B, Wang X, Zeng Z, Huang Z, Zhang L, et al. Expression signature, prognosis value, and immune characteristics of Siglec15 identified by pan-cancer analysis. Oncoimmunology. (2020) 9:1807291. doi: 10.1080/2162402x.2020.1807291

48. Yu Y, Blokhuis B, Diks M, Keshavarzian A, Garssen J, Redegeld F. Functional inhibitory siglec-6 is upregulated in human colorectal cancer-associated mast cells. Front Immunol. (2018) 9:2138. doi: 10.3389/fimmu.2018.02138

49. Kardava L, Moir S, Wang W, Ho J, Buckner C, Posada J, et al. Attenuation of HIV-associated human B cell exhaustion by siRNA downregulation of inhibitory receptors. J Clin Invest. (2011) 121:2614-24. doi: 10.1172/jci45685

50. Dijkman $\mathrm{R}$, van Doorn $\mathrm{R}$, Szuhai $\mathrm{K}$, Willemze R, Vermeer $\mathrm{M}$, Tensen C. Gene-expression profiling and array-based CGH classify CD4+CD56+ hematodermic neoplasm and cutaneous myelomonocytic leukemia as distinct disease entities. Blood. (2007) 109:1720-7. doi: 10.1182/blood-2006-04-018143

51. Chng W, Remstein E, Fonseca R, Bergsagel P, Vrana J, Kurtin $\mathrm{P}$, et al. Gene expression profiling of pulmonary mucosaassociated lymphoid tissue lymphoma identifies new biologic insights with potential diagnostic and therapeutic applications. Blood. (2009) 113:635-45. doi: 10.1182/blood-2008-02-14 0996

52. Tuscano J, Kato J, Pearson D, Xiong C, Newell L, Ma Y, et al. CD22 antigen is broadly expressed on lung cancer cells and is a target for antibody-based therapy. Cancer Res. (2012) 72:5556-65. doi: 10.1158/0008-5472.Can-120173
53. Pop L, Barman S, Shao C, Poe J, Venturi G, Shelton J, et al. A reevaluation of CD22 expression in human lung cancer. Cancer Res. (2014) 74:26371. doi: 10.1158/0008-5472.Can-13-1436

54. Kobayashi H, Boelte K, Lin P. Endothelial cell adhesion molecules and cancer progression. Curr Med Chem. (2007) 14:377-86. doi: 10.2174/092986707779941032

55. Mitra N, Banda K, Altheide T, Schaffer L, Johnson-Pais T, Beuten J, et al SIGLEC12, a human-specific segregating (pseudo)gene, encodes a signaling molecule expressed in prostate carcinomas. J Biol Chem. (2011) 286:2300311. doi: 10.1074/jbc.M111.244152

56. Barkal A, Brewer R, Markovic M, Kowarsky M, Barkal S, Zaro B, et al. CD24 signalling through macrophage Siglec-10 is a target for cancer immunotherapy. Nature. (2019) 572:392-6. doi: 10.1038/s41586-019-1456-0

57. Yamanaka M, Kato Y, Angata T, Narimatsu H. Deletion polymorphism of SIGLEC14 and its functional implications. Glycobiology. (2009) 19:8416. doi: $10.1093 /$ glycob/cwp052

58. Angata T, Ishii T, Motegi T, Oka R, Taylor R, Soto P, et al. Loss of Siglec-14 reduces the risk of chronic obstructive pulmonary disease exacerbation. Cell Mol Life Sci. (2013) 70:3199-210. doi: 10.1007/s00018-013-1311-7

59. Malik M, Chiles J, Xi H, Medway C, Simpson J, Potluri S, et al. Genetics of CD33 in Alzheimer's disease and acute myeloid leukemia. Hum Mol Genet. (2015) 24:3557-70. doi: 10.1093/hmg/ddv092

60. Lamba J, Pounds S, Cao X, Downing J, Campana D, Ribeiro R, et al. Coding polymorphisms in CD33 and response to gemtuzumab ozogamicin in pediatric patients with AML: a pilot study. Leukemia. (2009) 23:4024. doi: 10.1038/leu.2008.185

61. Khan N, Hills R, Virgo P, Couzens S, Clark N, Gilkes A, et al. Expression of CD33 is a predictive factor for effect of gemtuzumab ozogamicin at different doses in adult acute myeloid leukaemia. Leukemia. (2017) 31:105968. doi: 10.1038/leu.2016.309

62. Estus S, Shaw B, Devanney N, Katsumata Y, Press E, Fardo D. Evaluation of CD33 as a genetic risk factor for Alzheimer's disease. Acta Neuropathol. (2019) 138:187-99. doi: 10.1007/s00401-019-02000-4

63. Bremnes R, Al-Shibli K, Donnem T, Sirera R, Al-Saad S, Andersen S, et al. The role of tumor-infiltrating immune cells and chronic inflammation at the tumor site on cancer development, progression, and prognosis: emphasis on non-small cell lung cancer. J Thorac Oncol. (2011) 6:82433. doi: 10.1097/JTO.0b013e3182037b76

64. Merlo A, Dalla Santa S, Dolcetti R, Zanovello P, Rosato A. Reverse immunoediting: when immunity is edited by antigen. Immunol Lett. (2016) 175:16-20. doi: 10.1016/j.imlet.2016.04.015

65. Ohnishi K, Yamaguchi M, Erdenebaatar C, Saito F, Tashiro H, Katabuchi $\mathrm{H}$, et al. Prognostic significance of CD169-positive lymph node sinus macrophages in patients with endometrial carcinoma. Cancer Sci. (2016) 107:846-52. doi: 10.1111/cas.12929

66. Ohnishi K, Komohara Y, Saito Y, Miyamoto Y, Watanabe M, Baba H, et al. CD169-positive macrophages in regional lymph nodes are associated with a favorable prognosis in patients with colorectal carcinoma. Cancer Sci. (2013) 104:1237-44. doi: 10.1111/cas.12212

67. Saito Y, Ohnishi K, Miyashita A, Nakahara S, Fujiwara Y, Horlad H, et al. Prognostic significance of CD169+ lymph node sinus macrophages in patients with malignant melanoma. Cancer Immunol Res. (2015) 3:135663. doi: 10.1158/2326-6066.Cir-14-0180

68. Asano T, Ohnishi K, Shiota T, Motoshima T, Sugiyama Y, Yatsuda J, et al CD169-positive sinus macrophages in the lymph nodes determine bladder cancer prognosis. Cancer Sci. (2018) 109:1723-30. doi: 10.1111/cas.13565

69. Läubli H, Borsig L. Altered cell adhesion and glycosylation promote cancer immune suppression and metastasis. Front Immunol. (2019) 10:2120. doi: 10.3389/fimmu.2019.02120

70. Wculek S, Cueto F, Mujal A, Melero I, Krummel M, Sancho D. Dendritic cells in cancer immunology and immunotherapy. Nat Rev Immunol. (2020) 20:7-24. doi: 10.1038/s41577-019-0210-z

71. Shurin M, Gregory M, Morris J, Malyguine A. Genetically modified dendritic cells in cancer immunotherapy: a better tomorrow? Expert Opin Biol Ther. (2010) 10:1539-53. doi: 10.1517/14712598.2010.526105

72. Perez C, De Palma M. Engineering dendritic cell vaccines to improve cancer immunotherapy. Nat commun. (2019) 10:5408. doi: 10.1038/s41467-019-13368-y 
73. Shen M, Kang Y. Complex interplay between tumor microenvironment and cancer therapy. Front Med. (2018) 12:426-39. doi: 10.1007/s11684-018-0663-7

74. Bour-Jordan H, Blueston J. CD28 function: a balance of costimulatory and regulatory signals. J Clin Immunol. (2002) 22:1-7. doi: 10.1023/a:1014256417651

75. Slavik J, Hutchcroft J, Bierer B. CD28/CTLA-4 and CD80/CD86 families: signaling and function. Immunol Res. (1999) 19:124. doi: $10.1007 / \mathrm{bf} 02786473$

76. Solinas $\mathrm{C}, \mathrm{Gu}$-Trantien $\mathrm{C}$, Willard-Gallo K. The rationale behind targeting the ICOS-ICOS ligand costimulatory pathway in cancer immunotherapy. ESMO open. (2020) 5. doi: 10.1136/esmoopen-2019-000544

77. $\mathrm{Yu} \mathrm{X}$, Zheng $\mathrm{Y}$, Mao $\mathrm{R}$, Su Z, Zhang J. BTLA/HVEM signaling: milestones in research and role in chronic hepatitis $\mathrm{B}$ virus infection. Front Immunol. (2019) 10:617. doi: 10.3389/fimmu.2019.0 0617

78. Dougall W, Kurtulus S, Smyth M, Anderson A. TIGIT and CD96: new checkpoint receptor targets for cancer immunotherapy. Immunol Rev. (2017) 276:112-20. doi: 10.1111/imr.1 2518

79. Blake S, Dougall W, Miles J, Teng M, Smyth M. Molecular pathways: targeting CD96 and TIGIT for cancer immunotherapy. Clin Cancer Res. (2016) 22:5183-8. doi: 10.1158/1078-0432.Ccr-16-0933

80. Na H, Hudson S, Bochner B. IL-33 enhances Siglec-8 mediated apoptosis of human eosinophils. Cytokine. (2012) 57:16974. doi: 10.1016/j.cyto.2011.10.007
81. Hung J, Horn D, Woodruff K, Prihoda T, LeSaux C, Peters J, et al. Colonystimulating factor 1 potentiates lung cancer bone metastasis. Lab Invest. (2014) 94:371-81. doi: 10.1038/labinvest.2014.1

82. Ries C, Cannarile M, Hoves S, Benz J, Wartha K, Runza V, et al. Targeting tumor-associated macrophages with anti-CSF-1R antibody reveals a strategy for cancer therapy. Cancer Cell. (2014) 25:84659. doi: 10.1016/j.ccr.2014.05.016

83. Ries C, Hoves S, Cannarile M, Rüttinger D. CSF-1/CSF-1R targeting agents in clinical development for cancer therapy. Curr Opin Pharmacol. (2015) 23:45-51. doi: 10.1016/j.coph.2015.05.008

84. Schwardt O, Kelm S, Ernst B. SIGLEC-4 (MAG) antagonists: from the natural carbohydrate epitope to glycomimetics. Top Curr Chem. (2015) 367:151200. doi: 10.1007/128_2013_498

Conflict of Interest: The authors declare that the research was conducted in the absence of any commercial or financial relationships that could be construed as a potential conflict of interest.

Copyright (c) 2021 Zhang, Xie, Hu, Yu, Xie, Ye, Xu, Nian and Yuan. This is an open-access article distributed under the terms of the Creative Commons Attribution License (CC BY). The use, distribution or reproduction in other forums is permitted, provided the original author(s) and the copyright owner(s) are credited and that the original publication in this journal is cited, in accordance with accepted academic practice. No use, distribution or reproduction is permitted which does not comply with these terms. 